\title{
Giant Aggressive Extraocular Sebaceous Carcinoma: A Case Report
}

\author{
Fang Liu (iD \\ Fei Qi (iD \\ Xiuying Zhang \\ Mei Cao ii \\ Department of Dermatology, Beijing \\ Chaoyang Hospital, Capital Medical \\ University, Beijing, People's Republic of \\ China
}

\begin{abstract}
Sebaceous carcinoma (SC) is a rare, aggressive malignancy. Most lesions occur in the periocular region, with few cases presenting with extraocular lesions. Here, we report a case of an 89-year-old woman with a 3-month history of a rapidly growing giant extraocular SC. The diagnosis was based on skin biopsy findings. We advised the patient to undergo surgical excision of the carcinoma. However, the patient and her family rejected our proposed surgical treatment, opting for topical traditional Chinese medicine as alternative treatment. The patient subsequently died of unknown causes at home, four months after the first visit. This case of treatment failure emphasizes the importance of early detection and treatment for these aggressive tumors.
\end{abstract}

Keywords: sebaceous carcinoma, extraocular, skin neoplasms, malignancy, Muir-Torre syndrome

\section{Introduction}

Sebaceous carcinoma (SC) is an uncommon aggressive malignancy and accounts for $1-2$ cases per 1 million individuals per year. ${ }^{1} \mathrm{Up}$ to $70 \%$ of lesions arise from the preocular area. ${ }^{2}$ In this case report of a patient with SC, we describe the patient's clinical course and poor outcomes, likely due to treatment refusal.

\section{Case Presentation}

An 89-year-old woman presented to our clinic with a rapidly growing, painless mass on her left forehead, with no reported pruritus or burning sensations. The patient reported that the mass appeared unexpectedly three months ago, with no identified precipitating factors, and had grown rapidly. The patient denied the use of any medications and was otherwise asymptomatic, with no history of chronic diseases. No personal or family history of tumors was reported.

The mass was a 4-cm, protruding, ulcerated, fleshy, firm tumor with a moist and granular surface. Multiple scaly erythematous papules with yellowish crusts were observed on the margins of the lesion (Figure 1). A punch biopsy was performed to establish the diagnosis.

Histopathology demonstrated a basaloid neoplasm in lobules and sebaceous cells with vacuolated cytoplasm in the dermis under low power observation (magnification: $10 \times 10$ ) (Figure 2). In addition, ample coarse cells with atypical nuclei were found at high magnification (magnification: 40×10) (Figure 3). Immunohistochemical staining, including a positive epithelial membrane antigen (EMA) stain (Figure 4), confirmed sebaceous differentiation of the anaplastic cells, supporting the diagnosis of SC. As
Correspondence: Fang Liu

Department of Dermatology, Beijing Chaoyang Hospital, Capital Medical

University, Beijing, People's Republic of China

Tel +86- $|0-8523| 688$

$\mathrm{Fax}+86-10-85231217$

Email roseliufang@qq.com 


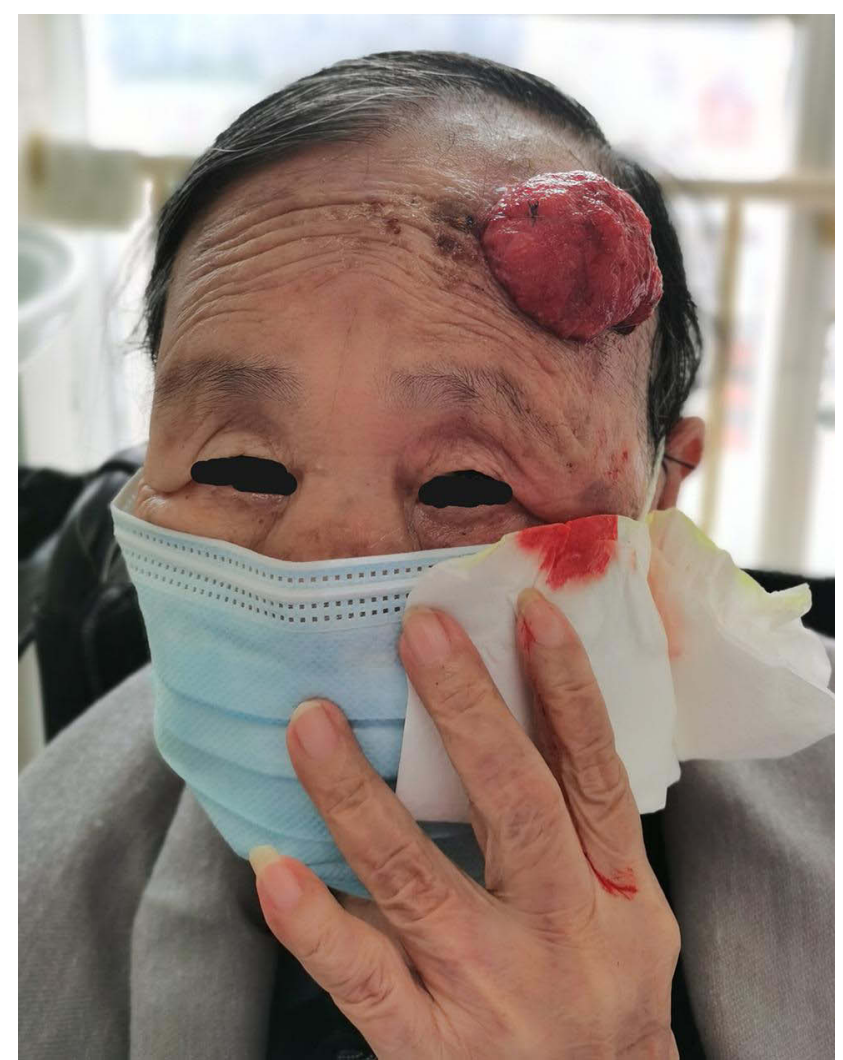

Figure I A 4-cm, protruding, ulcerated, fleshy, firm tumor with a moist and granular surface.

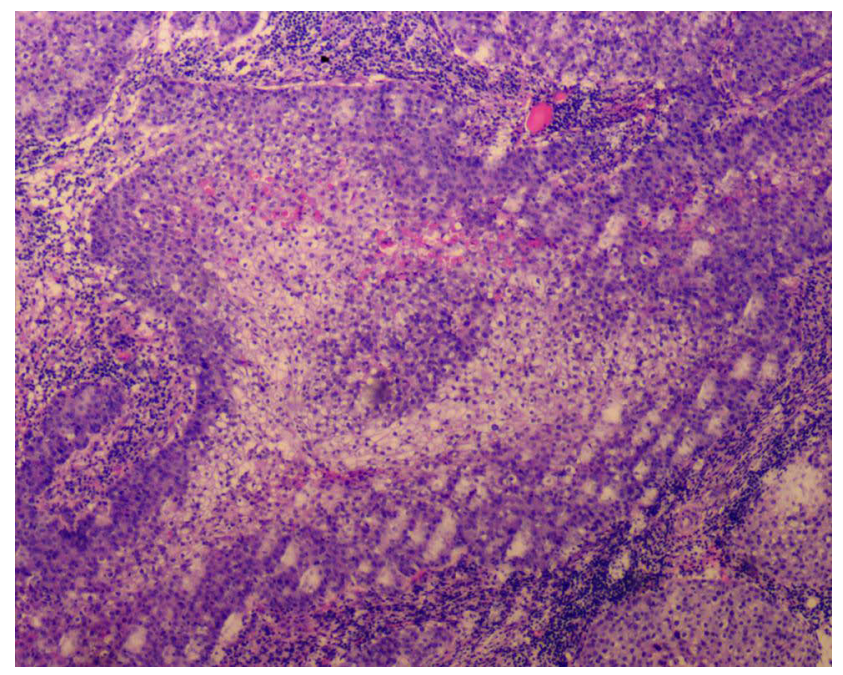

Figure 2 Basaloid neoplasm in lobules and sebaceous cells with vacuolated cytoplasm in the dermis (hematoxylin and eosin; magnification: 10×10).

recommended by recently published expert guidelines, ${ }^{3,4}$ we performed Mayo Muir-Torre syndrome (MTS) risk scoring at the first clinical visit after biopsy (score $=0$ ) to evaluate the need for genetic testing for loss of mismatch repair proteins, given the association between extraocular sebaceous carcinoma (ESC) and MTS.

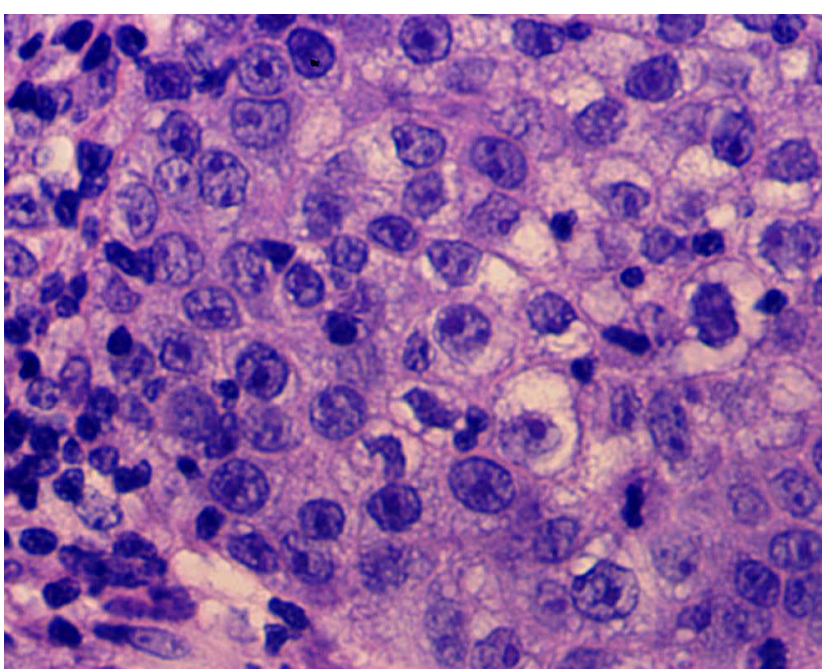

Figure 3 Ample coarse cells with atypical nuclei (magnification: $40 \times 10$ ).

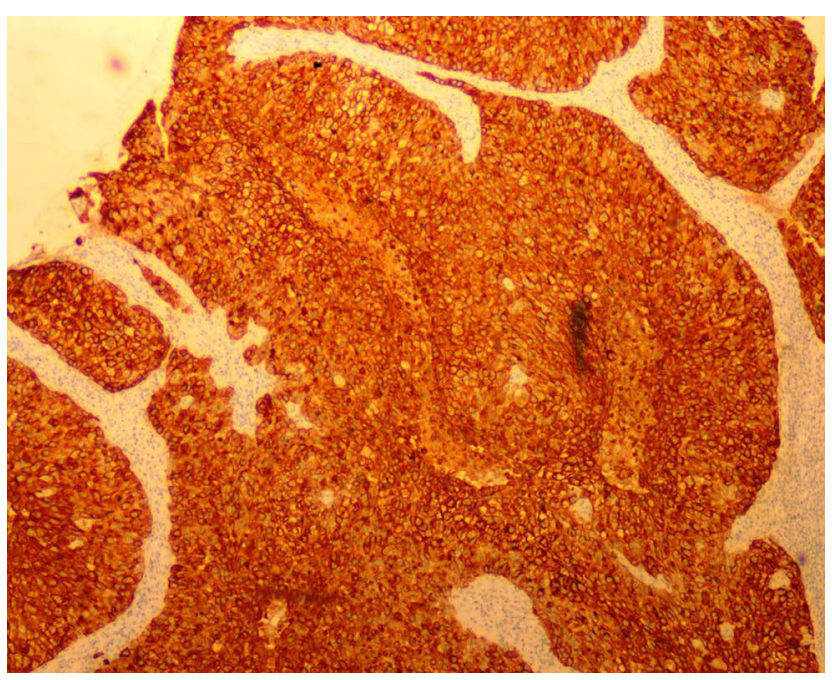

Figure 4 Positive immunohistochemical epithelial membrane antigen stain (magnification: $10 \times 10)$.

The patient and her family rejected the original surgical extirpation plan and requested topical traditional Chinese medicine as alternative therapy, which was unsuccessful. During the patient's last visit to our clinic, she complained of progressive tumor enlargement and infiltration. After neurosurgery and radiotherapy consultation, a radiation therapy plan was devised, in combination with laboratory tests and a highresolution computed tomography (CT) scan of the head. Unfortunately, the patient died of unknown causes before initiation of the planned tests and treatment (please see Figure 5:Timeline).

\section{Discussion}

$\mathrm{SC}$ is a rare, aggressive cutaneous malignancy of the sebaceous glands. ${ }^{3}$ It mostly affects the eyelid area 

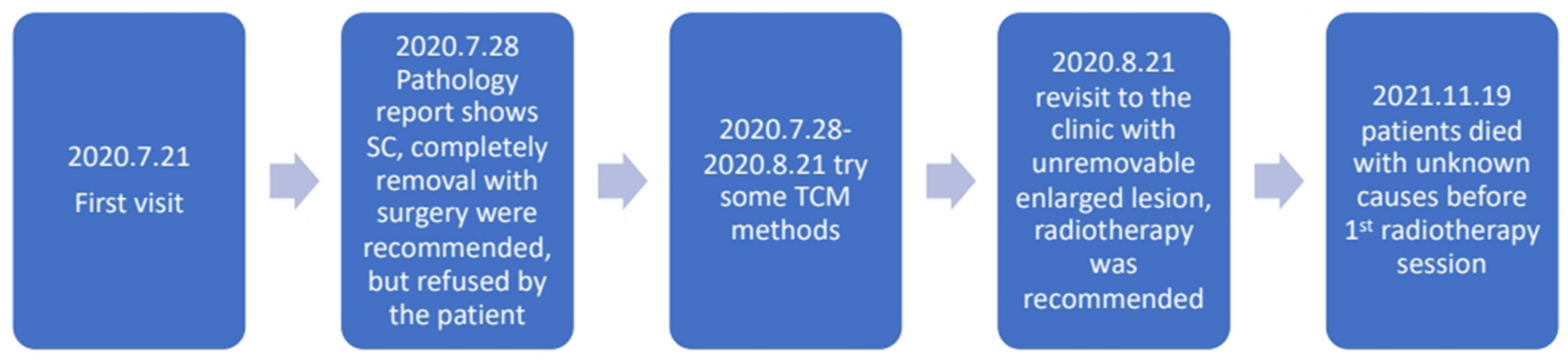

Figure 5 Timeline.

(periocular SC), especially the upper eyelids, given the high meibomian gland density. ${ }^{5}$ A minority of SC occurs on the skin of the head and neck (ESC), mostly in female patients. Other reported risk factors include Asian or South Asian race and advanced age. ${ }^{6}$

SCs can appear sporadically or may be linked to MTS, a variant of Lynch syndrome (hereditary nonpolyposis colorectal cancer syndrome), an autosomal dominant disorder characterized by a triad of single or multiple sebaceous neoplasms, keratoacanthomas, and internal malignancy. ${ }^{7}$ The Mayo MTS risk score, calculated by adding the four associated variables, has been used among patients with ESC to screen for MTS. ${ }^{4}$

ESC commonly presents as a painless, orange-to-tan ulcerated papule with a mean diameter of $1.4 \pm 0.3 \mathrm{~cm}$, and is commonly diagnosed 1-2 years after symptom onset. ${ }^{3}$ Dermoscopy is usually used to assist clinical diagnosis, despite limited diagnostic criterion. Cheng et al's analysis of the dermoscopic features of 15 cases of ESC versus other skin cancers found that the majority of ESCs $(66.67 \%)$ presented with polymorphic vessel patterns. ${ }^{8}$

Lesions are known to mimic the clinical features of other skin cancers such as basal cell carcinoma and squamous cell carcinoma, and histological samples are frequently obtained to aid diagnosis. ${ }^{9}$ Histological features of ESC include neoplastic basaloid cells in lobules or sheets with variable differentiation. Most ESCs demonstrate a tendency for dermal invasion and rarely invade the subcutaneous tissue and muscle. Immunohistochemistry markers such as nuclear factor XIIIa (clone AC-1A1 mouse monoclonal), EMA, androgen receptor, and adipophilin can aid diagnosis. ${ }^{3}$

$\mathrm{SC}$ is a locally aggressive tumor that can metastasize through local regional lymph nodes and distal sites. Regional lymph node palpation is necessary to evaluate for metastasis and stratify risk based on the eighth edition of the American Joint Committee on Cancer staging system. ${ }^{3}$ For those with stage T2c or higher, ultrasonography or CT is recommended for detecting lymphadenopathy. To detect distant metastases, CT or positron emission tomography-CT should be considered.

The main goal of localized SC treatment is complete tumor removal. Surgical excision with complete circumferential peripheral and deep margin assessment is considered the first-line therapy for ESC in the head and neck region. For patients with nodal metastases that are surgically irresectable, radiotherapy may be performed as the primary treatment. ${ }^{3}$

With growing interest in immunotherapy and an increasing understanding of the complex relationship between SC and the immune system, immune checkpoint blockade via PD-1/PD-L1 has been identified as a potential immunotherapeutic target. Pembrolizumab, a highly selective humanized monoclonal immunoglobulin antibody for PD-1, was used in several recurrent and advanced cases of SC with notable clinical responses. More clinical trials are needed to determine the effectiveness of pembrolizumab and other biologics in the treatment of advanced SC. ${ }^{10}$

In the present case, the lesion appeared rapidly and reached over $4 \mathrm{~cm}$ in diameter over a 3-month period. The lesion's size and invasiveness distinguishes it from other published cases of SC. ${ }^{11}$ Factors that may have contributed to the poor clinical outcome in the present case include the patient's advanced age, the patient's and her family's health beliefs and attitude towards surgical treatment, and delayed definitive treatment.

\section{Conclusion}

$\mathrm{SC}$, especially ESC, is a rare, highly invasive dermatological malignancy. Early diagnosis with complete surgical removal may achieve a good prognosis. Such cases need perioperative treatment monitoring. 


\section{Ethical Statement}

A signed consent was obtained from the family member for the publication of the case details accompanying images. Publication of the case details was approved by Beijing Chaoyang Hospital, Capital Medical University.

\section{Acknowledgments}

We would like to acknowledge Editage for English language editing.

\section{Disclosure}

The authors report no conflicts of interest in this work.

\section{References}

1. Knackstedt T, Samie FH. Sebaceous carcinoma: a review of the scientific literature. Curr Treat Options Oncol. 2017;18:47. doi:10.1007/s11864-017-0490-0

2. Orr CK, Yazdanie F, Shinder R. Current review of sebaceous cell carcinoma. Curr Opin Ophthalmol. 2018;29:445-450. doi:10.1097/ ICU.0000000000000505

3. Owen JL, Kibbi N, Worley B, et al. Sebaceous carcinoma: evidence-based clinical practice guidelines. Lancet Oncol. 2019;20: e699-e714. doi:10.1016/S1470-2045(19)30673-4
4. Roberts ME, Riegert-Johnson DL, Thomas BC, et al. A clinical scoring system to identify patients with sebaceous neoplasms at risk for the Muir-Torre variant of Lynch syndrome. Genet Med. 2014;16:711-716. doi:10.1038/gim.2014.19

5. Song A, Carter KD, Syed NA, et al. Sebaceous cell carcinoma of the ocular adnexa: clinical presentations, histopathology, and outcomes. Ophthalmic Plast Reconstr Surg. 2008;24(3):194-200. doi:10.1097/ IOP.0b013e31816d925f

6. Dasgupta T, Wilson LD, Yu JB. A retrospective review of 1349 cases of sebaceous carcinoma. Cancer. 2009;115:158-165. doi:10.1002/ cncr.23952

7. Dores GM, Curtis RE, Toro JR, et al. Incidence of cutaneous sebaceous carcinoma and risk of associated neoplasms: insight into Muir-Torre syndrome. Cancer. 2008;113:3372-3381. doi:10.1002/ cncr.23963

8. Cheng CY, Su HJ, Kuo TT. Dermoscopic features and differential diagnosis of sebaceous carcinoma. J Dermatol. 2020;47:755-762. doi:10.1111/1346-8138.15384

9. Jakobiec FA, Mendoza PR. Eyelid sebaceous carcinoma: clinicopathologic and multiparametric immunohistochemical analysis that includes adipophilin. Am J Ophthalmol. 2014;157:186-208 e2. doi:10.1016/j.ajo.2013.08.015

10. Lin AC, Shriver EM. The role of pembrolizumab in the treatment of sebaceous carcinoma. Int Ophthalmol Clin. 2020;60:39-46. doi:10. 1097/IIO.0000000000000309

11. Aung PP, Batrani M, Mirzabeigi M, et al. Extraocular sebaceous carcinoma in situ: report of three cases and review of the literature. J Cutan Pathol. 2014;41:592-596. doi:10.1111/cup.12 351

\section{Publish your work in this journal}

Clinical, Cosmetic and Investigational Dermatology is an international, peer-reviewed, open access, online journal that focuses on the latest clinical and experimental research in all aspects of skin disease and cosmetic interventions. This journal is indexed on CAS.
The manuscript management system is completely online and includes a very quick and fair peer-review system, which is all easy to use. Visit http://www.dovepress.com/testimonials.php to read real quotes from published authors. 